\title{
Study of the interaction between roof bolting of the output and a breed array inclined to creep
}

\author{
Mikhail Pleshko, ${ }^{1, *}$, Boris Cartozia ${ }^{1}$ and Maxim Podmiglazov ${ }^{2}$ \\ ${ }^{1}$ National University of Science and Technology MISIS (Moscow Institute of Steel and Alloys), \\ 119991, Leninskij Av., 4, Moskow, Russia \\ ${ }^{2}$ VostokCoal Management Company, 143084, estate 100, "Residence on Rublevka" Business Center, \\ Usovo, Odinstovsky District, Moscow Region, Russia
}

\begin{abstract}
The basic equations of the theory of linear hereditary creep are considered. An algorithm for determining the value of the anchor tension as a result of deformation of rocks in the bottom-hole zone of the barrel is presented. The area of the scope of roof bolting trunks in various breeds is defined.
\end{abstract}

\section{Description of the method of calculation}

Consider the interaction between roof bolting in the bottom-hole zone of the barrel and a breed array that exhibits the properties of creep [1-4].

Apply the theory of linear hereditary creep proposed by L. Boltzmann and modified by Volterr.

The creep of rocks is described by the Voltaire integral equation of the second kind

$$
\varepsilon(t)=\frac{\sigma_{0}}{E}+\frac{1}{E} \int_{0}^{t} L(t-\tau) \cdot \sigma(t) d \tau,
$$

where $\sigma(\mathrm{t}), \varepsilon(\mathrm{t})$ - are stresses and strains at time $\mathrm{t}$;

$\tau$ - time before instant $\mathrm{t}$;

$L(t-\tau)$ - the influence function (creep kernel).

At constant stresses from the equation (2.53) we obtain

$$
\varepsilon(t)=\frac{\sigma_{0}}{E}\left(1+\int_{0}^{t} L(\tau) d \tau\right) .
$$

Academician $\mathrm{Zh}$. S. Yerzhanov showed that the deformation of rocks to a certain level of loading corresponds to the equation (1) with the core of Abel type

$$
\varepsilon(t)=\frac{\sigma_{0}}{E}\left(1+\frac{\delta \cdot t^{1-\alpha}}{1-\alpha}\right),
$$

where $\delta, \alpha$-creep characteristics obtained experimentally.

Academician Rabotnov U. N. showed that the problem of linear hereditary creep theory can be formally considered as the problem of elasticity theory, in which instead of elastic

\footnotetext{
* Corresponding author: mixail-stepan@ mail.ru
} 
constants it is necessary to use temporal operators with creep kernel. Then the equation (2) can be represented as

$$
\varepsilon(t)=\frac{\sigma_{0}}{\bar{E}}
$$

where $\bar{E}$ - temporal operator.

Prof. Linkov, A. M. and Ph. D. of engineering Amusin B. Z. proved that in the tasks of mechanics of underground structures in which boundary conditions and volume forces are time independent, the operator expressions for elastic constants can be replaced by algebraic expressions corresponding to the kernel of the integral equation (the method of variable modules).

Based on the above, the creep equation (3) looks like:

$$
\varepsilon(t)=\frac{\sigma_{0}}{E}(1+\Phi)
$$

where $\Phi-$ the creep function:

$$
\Phi=\left(1+\frac{\delta \cdot t^{1-\alpha}}{1-\alpha}\right) .
$$

The time functions for the deformation modulus, shear modulus, and Poisson's ratio are: The time functions for the deformation modulus, shear modulus, and Poisson's ratio are:

$$
\begin{aligned}
E_{t} & =\frac{E}{1+\Phi} ; \\
G_{t} & =\frac{G}{1+\frac{3 \Phi}{2(1+v)}} ; \\
v_{t} & =0,5-\frac{0,5-v}{1+\Phi} .
\end{aligned}
$$

In the framework of the theory described above, we consider the interaction of anchors installed without tension from the bottom of the barrel, with the breed array.

As the bottom of the barrel moves away, the change in the stress-strain state of the massif is described by the expression $\alpha^{*} \lambda \gamma H$.

The coefficient $\alpha^{*}$, taking into account the lag of the considered point from the bottom, can be determined from the ratio [2-4]

$$
\alpha^{*}=1-\frac{u_{0}}{u_{\infty}}=\exp \left(-1,3 \frac{l}{r_{0}}\right),
$$

where $u_{0}$ - the starting array offset;

$u_{\infty}$ - the full array offset;

$l$ - distance from the considered point to the bottom of the trunk;

$r_{0}-$ the radius of the barrel is rough;

Hence we get

$$
\frac{u_{0}}{u_{\infty}}=\tilde{u}=1-\exp \left(-1,3 \frac{l}{r_{0}}\right) .
$$

The proportion of increment of the total coefficient $\alpha^{*}$ at each time $t$, defining the distance 1 , will be

$$
\Delta \alpha^{*}=\tilde{u}\left(t_{i+1}\right)-\tilde{u}\left(t_{i}\right) .
$$

Then the total value of the coefficient $\alpha^{*}$ can be expressed as 


$$
\alpha^{*}=\sum_{1}^{n} \Delta \alpha^{*} \text {. }
$$

To determine the tension of the anchors caused by the movement of the array, we use the analytical method for calculating the support developed By D. I. Kolin.

Anchor tension is equal to:

$$
F_{a}=B_{a} \frac{\Delta_{r}}{1+B_{a} K_{a}},
$$

$\Delta_{r}$ - the relative radial displacements of array points in case of unsupported excavation corresponding to the anchor ends;

$B_{a}$ - characterization of the anchor stiffness

$$
B_{a}=\frac{E_{a} A_{a}}{l},
$$

$E_{a}$ - the modulus of elasticity of the anchor material;

$A_{a}$ - anchor cross-sectional area;

$l$ - length of the anchor;

$K_{a}$ - coefficient of mutual influence of anchors

$$
K_{a}=K_{i i}+4 K_{i j},
$$

$K_{i i}$ - the coefficient of the effect of the efforts of the $F a$, applied to the array at the ends of the $\mathrm{i}$-th anchor, the relative displacement of the rocks on the ends of the given (i-th anchor); $K_{i j}$ - the coefficient of the effect of the efforts of the $F a$, applied to the array at the ends adjacent the j-th anchor, the relative displacement of the rocks on the ends of the $\mathrm{i}$-th anchor. Radial displacement of the barrel surface will be caused by the movement of the bottom and creep of rocks.

At each time moment t corresponding to a certain time of the tunneling cycle and the position of the bottom, the relative displacements will be

$$
\Delta_{r n}=\Delta_{r n}^{\prime}+\Delta_{r n}^{\prime \prime}
$$

$\Delta_{r i}^{\prime}$ - the proportion of relative displacements of the array points corresponding to the ends of the anchors caused by the removal of the bottom from the considered cross-section of the barrel for each approach

$$
\Delta_{r n}^{\prime}=\lambda \gamma H r_{0} \frac{\bar{l}}{1+\bar{l}} \cdot \frac{\Delta \alpha^{*}}{2 G_{t}}
$$

$\Delta_{r i}^{\prime}$ - the proportion of relative displacements of the array points corresponding to the ends of the anchors caused by the creep of rocks for a period equal to the duration of the tunneling cycle

$$
\Delta_{r n}^{\prime \prime}=\lambda \gamma H r_{0} \frac{\bar{l}}{1+l} \cdot \frac{\alpha^{*}}{2} \cdot\left(\frac{1}{G_{t n}}-\frac{1}{G_{t n-1}}\right),
$$

where $\bar{l}=l / r_{0}$.

Coefficients of influence in each of the considered points in time and the position of the bottom is equal

$$
K_{i i}=\frac{1}{2 G_{t n} l}\left[\left(1-v_{t n}\right) \frac{l}{r_{s}}-2 \frac{1-v_{t n}^{2}}{\pi^{2}}+\frac{l^{*}}{\pi\left(2 l^{*}-1\right)}\left(l^{*}+0,5-v_{t n}+\frac{1}{4\left(1-v_{t n}\right)} \cdot \frac{l^{*}-1}{\left(2 l^{*}-1\right)^{2}}\right)\right]
$$




$$
K_{i j}=\frac{1-v_{t n}}{2 \pi G_{t n} l}\left[1+\frac{3-4 v_{t n}}{8\left(1-v_{t n}\right)^{2}}\right] \cdot\left(l^{\prime}-\frac{1}{\sqrt{\left(l^{\prime}\right)^{2}+1}}\right),
$$

where $r_{\mathrm{s}}-$ the radius of the bearing washer;

$l^{*}=l / l_{\mathrm{z}}$;

$l_{\mathrm{z}}-$ the length of the fixed part of the anchor;

$l '=1 / a_{i j}$;

$a_{i j}$ - distance from the $\mathrm{j}$-th anchor to the anchor.

\section{Calculation results}

On the basis of the considered algorithm, the author determined the maximum application area of rigid anchors for three types of rocks (table. 1), for anchorage for the most frequently used parameters in practice: $l=1,8-2,2 \mathrm{~m} ; A_{a}=3,14 \mathrm{sm}^{2}$ (the diameter of the rod $20 \mathrm{~mm}$ ); $l_{z}=100 \mathrm{sm}$ type of reinforcing steel used $-\mathrm{A} 300, \mathrm{~A} 400$. The calculation was made for the barrel diameter of approximately $7.5 \mathrm{~m}$ with standard parameters of the tunneling cycle.

Table 1. Characteristics of rocks.

\begin{tabular}{|c|c|c|c|c|}
\hline No & The name of the rock & Poisson's ratio & $\alpha$ & $\delta, \mathrm{c}^{\alpha-1}$ \\
\hline 1 & Mudstones & 0.36 & 0.710 & 0.008 \\
\hline 2 & Siltstones & 0.34 & 0.726 & 0.0094 \\
\hline 3 & Sandstones & 0.31 & 0.670 & 0.0021 \\
\hline
\end{tabular}

As a condition of the anchor strength, the excess of the obtained rod tension value of the maximum design load on this type of anchor is accepted [6-9]:

$$
P_{\max }=S_{r} \cdot R_{p} \cdot m_{c},
$$

where $S_{r}-$ rod area, $\mathrm{m}^{2}$;

$R_{p}$ - the calculated resistance of the rod material to tension for reinforcing steel of class A300 $-R_{p}=270 \mathrm{MPa}, \mathrm{A} 400-R_{p}=340 \mathrm{MPa}$;

$m_{c}-$ the working condition ratio, in dry wells $m_{y}=0,9$; in wet wells $m_{y}=0,7-0,8$.

The field of application is presented in the form of graphs in Fig. 1 - 3 .

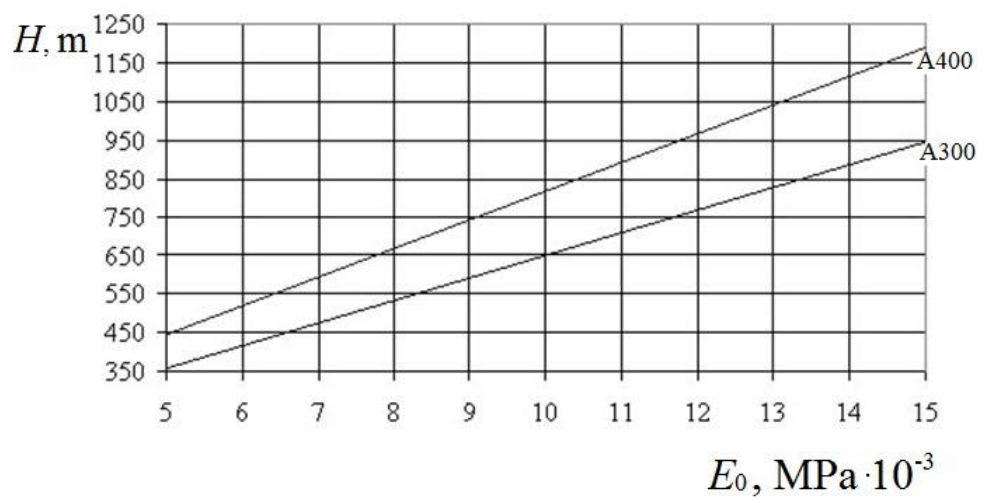

Fig. 1. The maximum application area of rigid anchors in mudstones. 


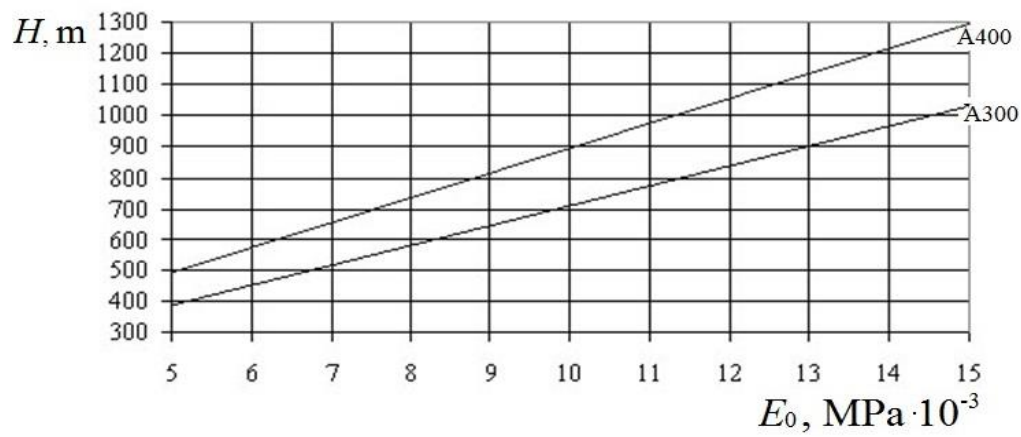

Fig. 2. The maximum application area of rigid anchors in siltstones.

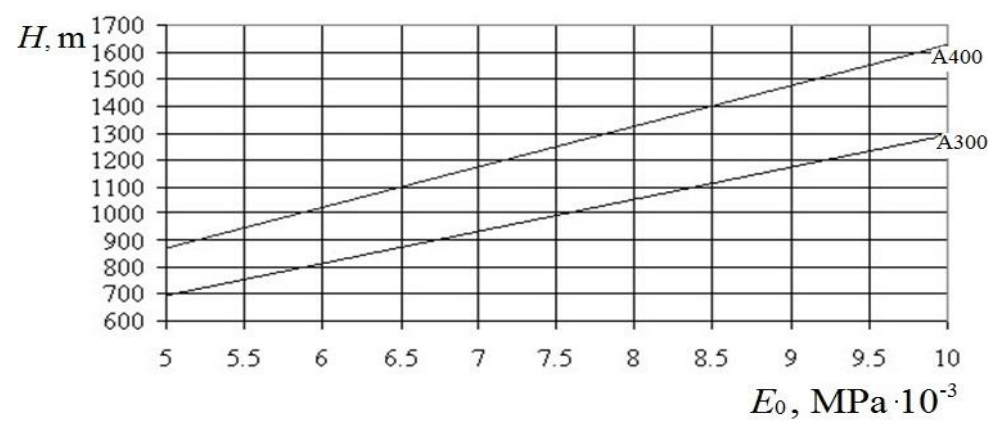

Fig. 3. The maximum application area of rigid anchors in sandstones.

\section{Conclusions}

From the graphs it can be seen that the tendency of rocks to creep largely determines the application area of anchors. In sandstones practically in all range of mountain-geological conditions it is possible to carry out fastening by rigid designs of anchors. In creep-prone mudstones and siltstones in a wide field there is a need to increase the strength characteristics of the anchors or the use of pliable structures.

The final determination of the necessary compliance of anchors in rocks prone to creep should be made on the basis of the results of full-scale measurements of the values of radial displacements in the bottom of the barrel [5-7].

\section{References}

1. M. Pleshko, B. Meskhi, B., M. Pleshko, MATEC Web of Conferences, 170, 03023 (2018)

2. A. Pankratenko, M. Pleshko and A. Isaev, MATEC Web of Conferences, 7, 02026 (2018)

3. N.S. Boolychev, Mechanics of underground structures (Nedra, Moscow, 1994)

4. F.I. Yagodkin, A.Y. Prokopov, M.S. Pleshko, A.N. Pankratenko, IOP Conference Series: Earth and Environmental Science, 87(6), 062014 (2017)

5. Meskhi, M. Pleshko, Y. Buligin, L. Alexeenko, M. Molev, IOP Conference Series: Earth and Environmental Science, 90(1), 012217 (2017)

6. M. Pleshko, A. Pankratenko, A. Revyakin, E3S Web of Conferences, 33, 02036 (2018)

7. M. Pleshko, I. Voinov, A. Revyakin, MATEC Web of Conferences, 106, 05004 (2017) 